\title{
PHENOLIC COMPOUNDS FROM STEM OF BOERHAVIA ERECTA L. GROWING IN VIETNAM
}

\author{
Do Thi My Lien, Nguyen Thi My Dung, Nguyen Kim Phi Phung \\ University of Science, VNU-HCM \\ (Manuscript Received on March $05^{\text {th }}$, 2011, Manuscript Revised October 25 ${ }^{\text {th }}$, 2011)
}

ABSTRACT: Plants of Boerhavia genus (Nyctaginaceae family) are known to have medicinal properties as antidiabetic, immunomodulatory effect, anticonvulsant, antistress, adaptogenic, hepatoprotective activity. Rotenoids in plants of this genus are reported as a new class of potent breast cancer resistance protein inhibitors. ${ }^{[5]}$ Boerhavia erecta L. was used in traditional African medicine but there are still no scientific research in Vietnam where it is widely grown. In this paper, we report the isolation of four compounds: 2,6-dimethoxybenzoquinone (1), (+)-catechin (2), isorhamnetin 3-O- $\beta$ D-glucopyranoside (3) and rutin (4). The structure of these compounds were determined through the interpretation of their MS and 1D, 2D-NMR data. This is the first time that these compounds are isolated from Boerhavia erecta $L$.

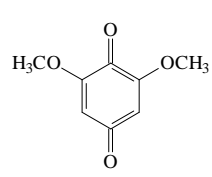

(1)

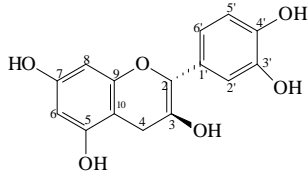

(2)

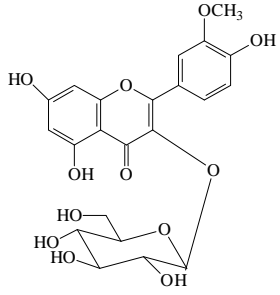

(3)

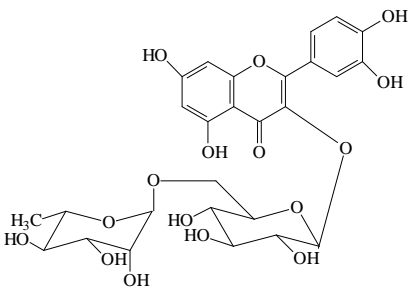

(4)

Key words: Boerhavia erecta L., benzoquinone, catechin, isorhamnetin, rutin.

\section{INTRODUCTION}

Boerhavia erecta L. (Nyctaginaceae) is an annual herb which is common in the tropics in both dry and rainy seasons and widely grown in Vietnam. The members of this genus were popular medicinal plants to treat several ailments such as malaria, hepatic disorders, jaundice, scanty urine, diabetic and anticancer. $^{[1]}$ 


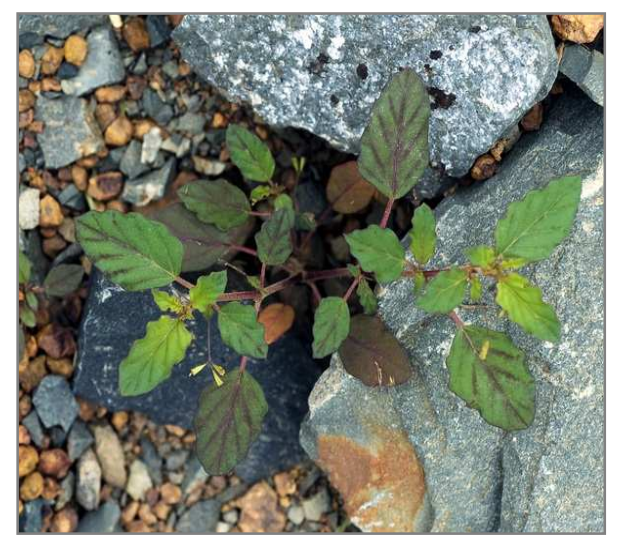

Figure 1. Boerhavia erecta L. in Viet Nam

This paper describes the isolation and structural elucidation of compounds: 2,6dimethoxybenzoquinone (1), (+)-catechin (2), isorhamnetin 3-O- $\beta$-D-glucopyranoside (3) and rutin (4).

\section{EXPERIMENTS}

\section{General experimental procedures}

The NMR spectra were recorded on Bruker Avance spectrometer at $500 \mathrm{MHz}$ for ${ }^{1} \mathrm{H}$ and $125 \mathrm{MHz}$ for ${ }^{13} \mathrm{C}$. All spectra were recorded in the Institute of Chemistry, Vietnam Academy of Science and Technology, Cau Giay Dist., Hanoi.

\section{Plant material}

Boerhavia erecta L. stems were collected at Thu Duc, Ho Chi Minh City, Vietnam in March 2009. The scientific name of the plant was identified by Pharmacist Phan Duc Binh. A voucher specimen (No USA 002) was deposited in the Herbarium of the Department of Organic Chemistry, Faculty of Chemistry, University of Science, National University - Ho Chi Minh City.

\section{Extraction and isolation}

Air-dried stems of $B$. erecta $(6.0 \mathrm{~kg})$ were extracted by maceration at room temperature and then evaporated in reduced pressure to give methanol residue $(850 \mathrm{~g})$. The residue was dissolved in methanol: water (1:9), then was partitioned against petroleum ether, ethyl acetate. The obtained solutions were evaporated to afford corresponding residues: petroleum ether (100 g), ethyl acetate (125 g) and the remaining methanol (630 g), respectively. The ethyl acetate extract (EA, 125 g) was silica gel column chromatographed to give 8 fractions. The column chrotomagraphy was applied on fraction 2 (3.5g) with chloroform as eluant to afford a yellow needle $(\mathbf{1}, 6 \mathrm{mg})$ and with chloroform-acetone (8:2) to give a white oil $(2,6 \mathrm{mg})$, on fraction 4 eluted with chloroform-methanol-water $(85: 15: 0.1)$ to afford a light yellow needle $(\mathbf{3}, 20 \mathrm{mg}$ ) and eluted with chloroform-methanol-water $(7: 3: 0.1)$ to afford a light yellow needle $(4,6 \mathrm{~g})$. 
2,6-Dimethoxybenzoquinone (1): yellow amorphous powder, the ${ }^{1} \mathrm{H}$ and ${ }^{13} \mathrm{C}-\mathrm{NMR}$ (chloroform- $d$ ) see Table 1

(+)-Catechin $\quad(2): \quad(2 R, 3 S)-2-(3,4-$ dihydroxyphenyl)-3,4-dihydro- $2 \mathrm{H}$-chromene3,5,7-triol, white oil, the ${ }^{1} \mathrm{H}$ and ${ }^{13} \mathrm{C}$-NMR (methanol- $d_{4}$ ) see Table 2 .

\section{Isorhamnetin 3-O-ק-D-glucopyranoside}

(3): yellow needle, the ${ }^{1} \mathrm{H}$ and ${ }^{13} \mathrm{C}-\mathrm{NMR}$ (pyridin- $d_{5}$ ) see Table 3 .

Rutin or quercetin $3-O-\alpha-\mathrm{L}-$ rhamnopyranosyl- $(1 \rightarrow 6)-O-\beta$-Dglucopyranoside (4): yellow needle, the ${ }^{1} \mathrm{H}$ and ${ }^{13} \mathrm{C}$-NMR (methanol- $d_{4}$ ) see Table 3 .

\section{RESULTS AND DISCUSSION}

Compound (1) was isolated as yellow needle $\left(6 \mathrm{mg}\right.$ ). The ${ }^{13} \mathrm{C}$-NMR spectrum (Table 1) of this compound showed 5 signals including two carbonyl carbons at $\delta 186.8$ and 176.6; two methine signals at $\delta 157.4$ and 107.4 and one methoxy group at $\delta 56.5$. The HSQC and HMBC experiments allowed the assignments of all protons and carbons resonances of (1) as 2,6dimethoxybenzoquinone.

Compound (2) was obtained as white oil (6 $\mathrm{mg}$ ). Its ${ }^{13} \mathrm{C}$-NMR spectrum (Table 2) showed 15 signals of a flavan-3-ol including seven aromatic quatenary carbons at $\delta 157.7,157.2$, 156.9, 145.6, 145.6 and 100.6, five aromatic methine carbon signals at $\delta 120.0,115.7$, 115.2, 96.1 and 95.4, two methine and one methylene carbon signals of a heterocyclic ring at $\delta 82.7,68.3$ and 28.8 , respectively. Its ${ }^{1} \mathrm{H}$ -
NMR spectrum showed two doublet signals at $\delta 6.91(J=1.5 \mathrm{~Hz}), \delta 6.80$

$\mathrm{Hz}) ;$ one doublet-doublet signal at $\delta 6.76$ $(J=1.5,8.0 \mathrm{~Hz})$ of protons of ring B; two doublet signals at $\delta 6.04(J=2.0 \mathrm{~Hz})$ and $\delta 6.89$ $(J=2.5 \mathrm{~Hz})$ of two meta-coupled protons of ring A. One doublet signal at $\delta 4.56(J=8.5$ $\mathrm{Hz}$ ) was assigned for proton $\mathrm{H}-2$; one multiplet signal for a methine group at $\delta 4.00$; and two doublet-doublet signals at $\delta 2.95$ and 2.53 were assigned for a methylene group $\mathrm{H}-4$; the signal at $\delta 2.95$ was overlapped by the solvent peak. The HSQC, HMBC experiments as well as the comparison with published data in the literature ${ }^{[2,7]}$ allowed the assignments of all protons and carbons resonances of (2) as (+)catechin. The NMR data of (2) was presented in Table 2.

Compound (3) was obtained as yellow needle $(20 \mathrm{mg})$. The ${ }^{13} \mathrm{C}-\mathrm{NMR}$ of $(\mathbf{3})$ spectrum (Table 3) showed 22 signals including of one carbonyl carbon, nine olefinic quatenary carbons, five aromatic methine carbons, one methoxy group, one anomeric carbon, four oxygenated methine carbons and one methylene group of a glucose unit. The ${ }^{1} \mathrm{H}$ NMR spectrum of (3) showed two doublet signals at $\delta 8.51(J=2.0 \mathrm{~Hz}), \delta 7.23(J=8.5$ $\mathrm{Hz})$; one doublet-doublet signals at $\delta 7.77(J=$ $2.0,8.5 \mathrm{~Hz}$ ) of three protons of ring $\mathrm{B}$; two doublet signals at $\delta 6.72(\mathrm{~J}=2.0 \mathrm{~Hz})$ and $\delta 6.70$ $(J=2.0 \mathrm{~Hz})$ of two meta-coupled protons of ring A; one doublet signal at $\delta 6.54(\mathrm{~J}=8.0 \mathrm{~Hz})$ (that was assigned for the anomeric proton) and one methoxy group at $\delta 3.59$. The data of 
HSQC and HMBC spectra showed the correlation between methoxy group and C-4' (149.4) so the aglycon moiety was isorharmnetin. The correlation between H-1", $\left(\delta_{\mathrm{H}} 6.53\right)$ and $\mathrm{C}-3\left(\delta_{\mathrm{C}} 134.8\right)$ confirmed that the sugar moiety linked to aglycon at its C-3. So (3) was elucidated as isorhamnetin 3-O- $\beta$-Dglucopyranoside. The NMR data of (3) was presented in Table 3.

Compound (4) was isolated as yellow needle. The ${ }^{13} \mathrm{C}-\mathrm{NMR}$ spectrum (Table 4) showed 27 signals including nine olefinic quatenary carbons at $\delta 166.0,163.0,159.3$, $158.5,149.8,145.8,135.6,123.1$, 105.6, five aromatic methine carbon signals at $\delta$ 125.6, 117.7, 116.1, 100.0, 94.9, two anomeric carbon signals at $\delta 104.7$ and 102.4, nine oxygenated methine carbon signals from $\delta 78.2$ to $\delta 68.6$ and one methyl group of rhamnose at $\delta$ 17.9. The ${ }^{1} \mathrm{H}$ NMR spectrum of (4) showed the characteristic signals at $\delta 5.12(1 \mathrm{H}, d, \mathrm{~J}=7.5$ $\mathrm{Hz}, \mathrm{Glc}-\mathrm{H}-1$ "') and $\delta 4.54(1 \mathrm{H}, d, \mathrm{~J}=1.0 \mathrm{~Hz}$, Rha-H-1',') ascribable to two anomeric protons. Additionally, the presence of 5,7disubstituted (ring A) and 3',4'-disubstituted (ring B) aromatic rings was suggested by the
${ }^{1} \mathrm{H}$ NMR data derived from the aglycon moiety: $\delta 7.69(1 \mathrm{H}, d, \mathrm{~J}=2.0 \mathrm{~Hz}, \mathrm{H}-2$ ') $7.64(1 \mathrm{H}, d d$, $\left.\mathrm{J}=2.0,8.5 \mathrm{~Hz}, \mathrm{H}-6^{\prime}\right), 6.89(1 \mathrm{H}, d, \mathrm{~J}=8.5 \mathrm{~Hz}$, H-5'), $6.42(1 \mathrm{H}, d, \mathrm{~J}=1.5 \mathrm{~Hz}, \mathrm{H}-8), 6.23(1 \mathrm{H}$, $d, \mathrm{~J}=1.5 \mathrm{~Hz}, \mathrm{H}-6)$. So, the aglycon moiety was quercetin. Using the COSY, HSQC and HMBC spectra, the connectivities of all the protons and carbons were determined. These spectroscopic data were suitable with the published ones ${ }^{[8]}$, so, (4) was elucidated as quercetin 3-rutinoside (Rutin). This compound was isolated at high content of $0.73 \%$ compared to the dried aerial part (quantitatively analyzed by HPLC method).

\section{CONCLUSION}

From Boerhavia erecta L., we obtained four phenolic compounds: 2,6dimethoxybenzoquinone (1), (+)-catechin (2), isorhamnetin 3-O- $\beta$-D-glucopyranoside (3) and rutin (4). This is the first time these compounds are known in this species. Literature showed that these flavones possess interesting biological activities so further studies are conducting.

Table 1. NMR data of compound (1)

\begin{tabular}{|c|c|c|c|}
\hline \multirow{2}{*}{ Position } & \multicolumn{3}{|c|}{ (1) $\left(\right.$ in $\left.\mathrm{CDCl}_{3}\right)$} \\
\cline { 2 - 4 } & $\delta_{\mathrm{H}}(\mathrm{J}$ in $\mathrm{Hz})$ & $\delta_{\mathrm{C}}$ & $\begin{array}{c}\mathrm{HMBC} \\
\left({ }^{1} \mathrm{H} \rightarrow{ }^{13} \mathrm{C}\right)\end{array}$ \\
\hline 1 & - & 186.8 & - \\
\hline 2 & - & 157.4 & $1,3,4,6$ \\
\hline 3 & $5.85 s$ & 107.4 & - \\
\hline 4 & - & 176.6 & $1,3,4,6$ \\
\hline 3 & $5.85 s$ & 107.4 & 2 \\
\hline 6 & - & 107.4 & 6 \\
\hline $2-\mathrm{OCH}_{3}$ & $3.82 s$ & 56.5 & - \\
\hline $6-\mathrm{OCH}_{3}$ & $3.82 s$ & 56.5 & \\
\hline
\end{tabular}


Table 2. NMR data of compound (2) in comparing with data in the literature ${ }^{[2,7]}$

\begin{tabular}{|c|c|c|c|c|c|}
\hline \multirow[b]{2}{*}{ Position } & \multicolumn{3}{|c|}{ (2) $\left(\right.$ in $\left.\mathrm{CD}_{3} \mathrm{OD}\right)$} & \multicolumn{2}{|c|}{ (+)-Catechin } \\
\hline & $\delta_{\mathrm{H}}(\mathrm{J}$ in $\mathrm{Hz})$ & $\delta_{\mathrm{C}}$ & $\begin{array}{c}\mathrm{HMBC} \\
\left({ }^{1} \mathrm{H} \rightarrow{ }^{13} \mathrm{C}\right)\end{array}$ & $\begin{array}{c}\delta_{\mathrm{H}}(\mathrm{J} \text { in } \mathrm{Hz}) \\
\left.\text { (in acetone- } d_{6}\right)\end{array}$ & $\begin{array}{c}\delta_{\mathrm{C}} \\
\text { ( in } \mathrm{CD}_{3} \mathrm{OD} \text { ) }\end{array}$ \\
\hline 2 & $4.57 d(8.5)$ & 82.7 & $3,4,1^{\prime}, 2^{\prime}, 6^{\prime}$ & $4.58 d(7.3)$ & 83.0 \\
\hline 3 & $4.00 \mathrm{~m}$ & 68.3 & - & $4.00 \mathrm{~m}$ & 68.9 \\
\hline 4 & $\begin{array}{c}2.95 m \\
2.53 d d(8.5,16.0)\end{array}$ & 28.8 & $2,3,10$ & $\begin{array}{l}2.95 d d(5.9,15.9) \\
2.52 d d(8.9,15.9)\end{array}$ & 28.6 \\
\hline 5 & - & 157.2 & - & - & 157.7 \\
\hline 6 & $6.04 d(2.0)$ & 96.2 & $7,8,10$ & $5.87 d(2.2)$ & 96.4 \\
\hline 7 & - & 157.7 & - & - & 157.9 \\
\hline 8 & $5.89 d(2.0)$ & 95.5 & $6,9,10$ & $6.01 d(2.2)$ & 95.6 \\
\hline 9 & - & 156.9 & - & - & 157.0 \\
\hline 10 & - & 100.6 & - & - & 100.9 \\
\hline 1 ' & - & 132.2 & - & - & 132.3 \\
\hline 2 & $6.91 d(1.5)$ & 115.2 & $2,1^{\prime}, 3^{\prime}, 6^{\prime}$ & 6.89 brs & 115.4 \\
\hline $3^{\prime}$ & - & 145.6 & - & - & 146.4 \\
\hline 4 ' & - & 145.6 & - & - & 146.3 \\
\hline 5 & $6.80 d(8.0)$ & 115.7 & $1^{\prime}, 3^{\prime}, 4^{\prime}$ & $6.90 d(8.0)$ & 116.2 \\
\hline $6{ }^{\prime}$ & $6.77 d d(1.5,8.0)$ & 120.0 & $2,2^{\prime}, 4^{\prime}$ & $6.84 d d(8.0,1.5)$ & 120.2 \\
\hline
\end{tabular}

Table 3. NMR data of compound (3) in comparing with data in the literature ${ }^{[6]}$

\begin{tabular}{|c|c|c|c|c|c|}
\hline \multirow[b]{2}{*}{ Position } & \multicolumn{3}{|c|}{ (3) ( in pyridin- $d_{5}$ ) } & \multicolumn{2}{|c|}{ Isorhamnetin 3-O- $\beta$-D-glucopyranoside } \\
\hline & $\delta_{\mathrm{H}}(\mathrm{J}$ in $\mathrm{Hz})$ & $\delta_{\mathrm{C}}$ & $\begin{array}{c}\text { HMBC } \\
\left({ }^{1} \mathrm{H} \rightarrow{ }^{13} \mathrm{C}\right)\end{array}$ & $\begin{array}{l}\delta_{\mathrm{H}}(\mathrm{J} \text { in } \mathrm{Hz} \text { ) } \\
\left(\text { in } \mathrm{CD}_{3} \mathrm{OD} \text { ) }\right.\end{array}$ & $\begin{array}{c}\delta_{\mathrm{C}} \\
\left(\text { in } \mathrm{DMSO}-d_{6}\right)\end{array}$ \\
\hline 2 & - & 157.6 & - & - & 156.3 \\
\hline 3 & - & 134.8 & - & - & 133.0 \\
\hline 4 & - & 178.7 & - & - & 177.3 \\
\hline 5 & - & 162.9 & - & - & 161.2 \\
\hline 6 & $6.70 d(2.0)$ & 99.8 & $7,8,10$ & $6.16 d(1.7)$ & 98.7 \\
\hline 7 & - & 165.9 & - & - & 165.1 \\
\hline 8 & $6.7 d(2.0)$ & 94.6 & $6,9,10$ & $6.37 d(1.7)$ & 93.7 \\
\hline 9 & - & 157.2 & - & - & 156.4 \\
\hline 10 & - & 105.3 & - & - & 103.9 \\
\hline
\end{tabular}


TẠP CHÍ PHÁT TRIỂN KH\&CN, TậP 14, SỐ T2 - 2011

\begin{tabular}{|c|c|c|c|c|c|}
\hline 1 ' & - & 122.1 & - & - & 121.0 \\
\hline 2 ' & $8.51 d(2.0)$ & 114.4 & $2,4^{\prime}, 6^{\prime}$ & $7.98 d(1.8)$ & 113.4 \\
\hline $3^{\prime}$ & - & 148.0 & - & & 146.9 \\
\hline $4^{\prime}$ & - & 151.2 & - & & 149.4 \\
\hline 5 & $7.23 d(8.5)$ & 116.2 & $1^{\prime}, 3^{\prime}$ & $6.89 d(8.4)$ & 115.1 \\
\hline $6^{\prime}$ & $7.77 d d(2.0,8.5)$ & 123.1 & $2^{\prime}, 4^{\prime}$ & $7.50 d d(1.8,8.4)$ & 121.9 \\
\hline 1 ', & $6.53 d(8.0)$ & 103.5 & 3 & $5.46 d(7.6)$ & 101.4 \\
\hline 2 ', & $4.31 \mathrm{~m}$ & 71.44 & 1 & $3.30 \mathrm{~m}$ & 74.3 \\
\hline 3, & $4.41 \mathrm{~m}$ & 76.3 & - & $3.45 t(8.8)$ & 76.6 \\
\hline 4 '” & $4.35 \mathrm{~m}$ & 79.0 & - & $3.41 t(8.8)$ & 70.3 \\
\hline 5, & $4.04 \mathrm{~m}$ & 78.5 & - & $3.30 \mathrm{~m}$ & 76.1 \\
\hline $6 "$ & $4.32 \mathrm{~m}, 4.40 \mathrm{~m}$ & 62.2 & - & $\begin{array}{r}3.37 d d \quad(8.5 \\
13.1)\end{array}$ & 67.3 \\
\hline $3^{\prime}-\mathrm{OCH}_{3}$ & $3.93 \mathrm{~s}$ & 56.2 & 3 ' & $3.84 s$ & 55.9 \\
\hline
\end{tabular}

Table 4. NMR data of compound (4) in comparing with data in the literature ${ }^{[3,8]}$

\begin{tabular}{|c|c|c|c|c|c|}
\hline \multirow[b]{2}{*}{ Position } & \multicolumn{3}{|c|}{ (4) $\left(\right.$ in $\left.\mathrm{CD}_{3} \mathrm{OD}\right)$} & \multicolumn{2}{|c|}{ Rutin } \\
\hline & $\delta_{\mathrm{H}}(\mathrm{J}$ in $\mathrm{Hz})$ & $\delta_{\mathrm{C}}$ & $\begin{array}{c}\text { HMBC } \\
\left({ }^{1} \mathrm{H} \rightarrow{ }^{13} \mathrm{C}\right)\end{array}$ & $\begin{array}{l}\delta_{\mathrm{H}}(\mathrm{J} \text { in } \mathrm{Hz}) \\
\left(\text { in } \mathrm{CD}_{3} \mathrm{OD}\right)\end{array}$ & $\begin{array}{c}\delta_{\mathrm{C}} \\
\left(\text { in } \mathrm{DMSO}-d_{6}\right)\end{array}$ \\
\hline 2 & - & 158.5 & - & - & 156.6 \\
\hline 3 & - & 135.6 & - & - & 133.5 \\
\hline 4 & - & 179.4 & - & - & 177.4 \\
\hline 5 & - & 163.0 & - & - & 161.3 \\
\hline 6 & $6.23 d(1.5)$ & 100.0 & $5,7,8,10$ & $6.11 d(2.0)$ & 99.0 \\
\hline 7 & - & 166.0 & - & - & 164.1 \\
\hline 8 & $6.42 d(1.5)$ & 94.9 & $6,7,9,10$ & $6.28 d(2.0)$ & 93.9 \\
\hline 9 & - & 159.3 & - & - & 156.8 \\
\hline 10 & - & 105.6 & - & - & 104.2 \\
\hline 1 ' & - & 123.1 & - & - & 121.4 \\
\hline $2^{\prime}$ & $7.69 d(2.0)$ & 117.7 & $2,3^{\prime}, 4^{\prime}, 6^{\prime}$ & $7.66 d(2.2)$ & 115.4 \\
\hline $3^{\prime}$ & - & 145.8 & - & - & 144.8 \\
\hline 4 & - & 149.8 & - & - & 148.5 \\
\hline 5 & $6.89 d(8.5)$ & 116.7 & $3^{\prime}, 4^{\prime}, 6^{\prime}$ & $6.85 d(8.4)$ & 116.5 \\
\hline 6 & $7.64 d d(2.0,8.5)$ & 123.6 & $2,2^{\prime}, 4^{\prime}$ & $7.63 d d(2.2,8.4)$ & 121.5 \\
\hline
\end{tabular}




\begin{tabular}{|c|c|c|c|c|c|}
\hline $1 "$, & $5.12 d(7.5)$ & 104.7 & $3,5^{\prime \prime}$ & $5.46 d(7.6)$ & 101.4 \\
\hline $2 "$ & - & 75.7 & - & $3.30 \mathrm{~m}$ & 74.3 \\
\hline $3 "$ & - & 78.2 & - & $3.45 t(8.8)$ & 76.6 \\
\hline $4 "$, & - & 72.1 & - & $3.41 t(8.8)$ & 70.3 \\
\hline 5, & - & 77.2 & - & $3.30 \mathrm{~m}$ & 76.1 \\
\hline $6 "$ & $3.81 d d(1.0,9.5)$ & 68.6 & - & $3.37 d d(8.5,13.1)$ & 67.3 \\
\hline $1 "$, & $4.54 d(1.0)$ & 102.4 & 6" & $4.47 d(1.5)$ & 100.9 \\
\hline $2, "$, & $3.65 d d(1.5,3.5)$ & 72.3 & - & $3.47 d d(1.5,3.4)$ & 7.06 \\
\hline $3, "$ & $3.55 d d(3.5,8.5)$ & 71.4 & - & $3.35 \mathrm{~m}$ & 70.6 \\
\hline 4",' & - & 73.9 & - & $3.16 \mathrm{~m}$ & 72.1 \\
\hline $5, "$ & - & 69.7 & - & $3.34 \mathrm{~m}$ & 68.5 \\
\hline $6 "$, & $1.13 d(6.5)$ & 17.9 & 4",',5", & $1.02 d(6.3)$ & 18.0 \\
\hline
\end{tabular}

\title{
CÁC HỢP CHẤT PHENOL TÙ๋ THÂN LÁ CÂY NAM SÂM ĐÚ'NG BOERHAVIA ERECTA L., HỌ BÔNG PHẤN (NYCTAGINACEAE)
}

\author{
Đỗ Thị Mỹ Liên, Nguyễn Thị Mỹ Dung, Nguyễn Kim Phi Phụng
}

Trường Đại học Khoa Học Tự Nhiên, ĐHQG-HCM

TÓM TÄT: Các loài thuộc chi Boerhavia ho Bông phấn (Nyctaginaceae) đã được sủ dụng ở một số nuớc trên thế giới làm thuốc chữa các bệnh về gan, thận, tiểu đường, tăng khả năng miễn dịch... Ở Việt Nam, cây Nam sâm đứng được sinh truởng và phân bố rất rộng rãi, tuy nhiên cây này chura đurợc nghiên cưu về thành phần hóa học. Bài báo này trình bày việc cô lập tù cây đuợc bốn hợp chất: 2,6dimethoxybenzoquinon (1), (+)-catechin (2), isorhamnetin 3-O- $\beta$-D-glucopyranosid (3) và rutin (4). Cấu trúc hóa học của các hợp chất trên được xác định bằng các phuoong pháp phổ nghiệm NMR và so sánh vói tài liệu tham khảo. Các hợp chát này lần đầu tiên cô lập đuợc tù̀ cây Nam sâm đúng.

Tù khóa: Boerhavia erecta L., benzoquinon, isorhamnetin, rutin. 


\section{REFERENCES}

[1]. Adesina S.K., Anticonvulsant properties of the roots of Boerhavia diffusa. Quarterly Journal of Crude Drug Research, 17, pp. 84-86 (1979)

[2]. Cecile Cren-Olive, Jean-Michel Wieruszeski, Emmanuel Maes and Christian Rolando, Catechin and epicatechin deprotonation followed by ${ }^{13} \mathrm{C}$ NMR, Tetrahedron Letters, 43, pp. 45454549 (2002).

[3]. Ernest Wenkert et al, Carbon-13 nuclear magnetic resonance spectroscopy of flavonoid and isoflavonoid compounds, Phytochemistry, 16, pp. 1811-1816 (1977)

[4]. Florian C. Stintzing et al, Betacyanins and phenolic compounds from Amaranthus spinosus L.and Boerhavia erecta L., Z. Naturforsch. 59c, pp. 1-8 (2004), http://www.znaturforsch.com/ac/v59c/s59c 0001.pdf

[5]. Francesca Borelli et al, Spasmolytic effects of nonprenylated rotenoid constituents of Boerhavia diffusa roots, J. Nat. Prod., 69, pp. 903-906 (2006).

[6]. Hichem Ben Salah et al, Flavonol triglycosides from the leaves of Hammada scoparia (Pomel)Iljin, Chem. Pharm. Bull. 50(9), pp. 1268-1270 (2002).

[7]. Romanczyk Jr., Preparation of (+)catechin, (-)-epicatechin, (-)-catechin, and (+)-epicatechin and their 5,7,3',4'-tetra- $O$ benzyl analogues, www.freepatentsonline.com.

[8]. Yana Abdullah, Bernd Schneider, Maike Petersen, Occurrence of rosmalinic acid, chlorogenic acid and rutin in Marantaceae species, Phytochemistry Letters, 1, pp. 199-203 (2008). 